\title{
Close Versus Decisive Victory Moderates the Effect of Testosterone Change on Competitive Decisions and Task Enjoyment
}

\author{
Pranjal H. Mehta • Nicholas A. Snyder • \\ Erik L. Knight • Bethany Lassetter
}

Received: 25 September 2014 / Revised: 17 October 2014 / Accepted: 27 October 2014 /

Published online: 6 November 2014

(C) Springer International Publishing 2014

\begin{abstract}
Prior research found that testosterone change after defeat predicted the decision to compete against the same opponent, but testosterone change after victory was unrelated to competitive behavior. The present research tested whether testosterone responses have differential effects on competitive decision-making depending on whether an individual either barely or decisively won a competition. Seventy-one undergraduate males provided an afternoon saliva sample and then participated in a laboratory cognitive contest in which they were randomly assigned to experience a relatively close or decisive victory against a male confederate. Participants provided a second saliva sample after the competition and then chose whether to: (a) compete against the same opponent, (b) compete against a new opponent, or (c) complete an alternative non-competitive task. Participants also reported how much they enjoyed the competitive task. Testosterone change and the propensity to compete were positively related after a decisive victory, but were negatively related after a close victory. These effects were driven by the decision to compete against a new opponent. In fact, very few participants chose to compete against the same opponent. Testosterone change after a decisive victory was also positively associated with participants' self-reported enjoyment of the competitive task. Together, these results provide new evidence that a close versus decisive victory moderates the effect of testosterone change on future competitive behavior, an effect that may be linked to changes in reward processing systems.
\end{abstract}

Keywords Testosterone $\cdot$ Cortisol $\cdot$ Decision-making $\cdot$ Competition $\cdot$ Victory $\cdot$ Reward

Testosterone influences status-seeking behaviors such as aggression and dominance in a variety of species (e.g., birds, Wingfield et al. 1990; fish, Oliveira et al. 2009; mice,

P. H. Mehta $(\square) \cdot$ N. A. Snyder $・$ E. L. Knight

Department of Psychology, University of Oregon, Eugene, OR 97403, USA

e-mail:mehta@uoregon.edu

B. Lassetter

Department of Psychology, University of Iowa, Iowa 52242-1407, USA 
Trainor et al. 2004), but the precise role of testosterone in human social behavior remains unclear. For example, meta-analyses reveal only a weak positive association between baseline testosterone and human aggression ( $r=.08$, Archer et al. 2005), and baseline testosterone is positively related to dominant behaviors in some human studies but not others (Archer 2006; Carré et al. 2011). Baseline testosterone is moderately stable when measured at the same time of day and is thought to tap into a person's chronic concern for status similar to a personality trait (Sellers et al. 2007). But testosterone concentrations also fluctuate in status-relevant social interactions, and these dynamic changes in testosterone may play a role in modulating social behaviors above and beyond baseline testosterone concentrations (Archer 2006; Carré et al. 2011; Mazur 1985; Mazur and Booth 1998).

Mazur's (1985) biosocial model of status (BMS) has been the primary theory that has guided research on the dynamic relationship between testosterone and status in humans (see also the challenge hypothesis for a similar theory based on research in birds, Wingfield et al. 1990). The BMS posits a reciprocal relationship between testosterone and status. According to the model, (i) testosterone should rise after victory and drop after defeat in status contests, and (ii) these changes in testosterone should in turn influence subsequent status-seeking behaviors. Specifically, the model predicts that a testosterone increase after victory should stimulate further attempts at gaining or maintaining high status, whereas a testosterone decrease after defeat should lead individuals to flee from further competition in order to avoid greater loss of status.

Many studies have tested the first prediction of the BMS that winners should increase in testosterone relative to losers (Archer 2006; Oliveira and Oliveira 2014). Although some studies have indeed shown this effect, other studies found nonsignificant differences, and still others showed the complete opposite pattern (Oliveira et al. 2013; Oliveira and Oliveira 2014; Zilioli et al. 2014). The large variation in testosterone response profiles across individuals and studies may be driven by psychological factors, but the precise psychological mechanisms remain unclear (see recent review, Oliveira and Oliveira 2014). Together, the evidence suggests that there is substantial variability in testosterone responses that cannot be explained by competition outcome alone.

Far fewer studies in humans have tested the second prediction of the BMS that post-competition testosterone should influence subsequent status-seeking behaviors (for a relevant review, see Carré et al. 2011). Mehta and Josephs (2006) conducted the first empirical study in humans that examined the relationship between testosterone change from before to after competition and subsequent social behavior. In this study, sixty-four undergraduate males were invited to the lab in dyads. The two men competed on a Number Tracking Task (NTT) that was ostensibly an intelligence measure of "spatial processing speed". In reality, participants were randomly assigned to win or lose by giving the chosen winner an easier version of the task than the chosen loser. Participants provided pre- and post-competition saliva samples to measure testosterone changes. After providing the post-competition saliva sample, participants were asked to choose the next experimental task. They could choose to (a) compete against the same participant on a new version of the NTT, or (b) complete a non-competitive task that would take about as long as the NTT (a questionnaire on food, music, and entertainment preferences). The results showed variation in testosterone change that was not explained by competition outcome. Consistent with the BMS's 
prediction that testosterone responses should encourage subsequent attempts at gaining status, losers who increased in testosterone were more likely to choose to compete against the same opponent than losers who decreased in testosterone. However, there was a null association between testosterone change and decisions to compete in winners (see also Carré et al. 2009 for a similar pattern of results on a measure of aggressive behavior). The present research was designed to build upon the Mehta and Josephs (2006) study in order to clarify the relationship between post-victory testosterone changes and subsequent competitive decisions.

We propose two possible explanations for these prior null results in winners. First, Mehta and Josephs (2006) offered participants the choice to compete against the same opponent. The BMS proposes that testosterone increases after victory should encourage future attempts at gaining or protecting high status, but competing against the same opponent (the loser) does not offer the winner an opportunity to rise further in the hierarchy because the winner has already gained status over the other opponent. Instead, increases in testosterone after winning may guide future decisions to compete against a new opponent. To test this possibility, the present study used the same two options that participants in Mehta and Josephs (2006) were offered (compete against the same opponent, complete a non-competitive task) but added a third option in which participants could choose to compete against a new opponent on the NTT.

Second, Mehta and Josephs (2006) used real dyads to study testosterone responses and behavior, but this research design creates heterogeneity across participants in the victory experience. The association between post-victory testosterone change and competitive decisions may depend on status-relevant contextual factors, such as whether the victory is close or decisive. Decisive victories model stable status hierarchies in which the winner clearly dominates the loser, whereas close victories model unstable hierarchies in which the winner's higher status position is uncertain. Testosterone increases may stimulate the desire to compete against a new opponent only after a decisive victory as a strategy to rise further in the status hierarchy. This prediction is generally consistent with the BMS, which implies that a potent boost in status (i.e., a decisive victory experience) coupled with a testosterone increase should encourage future status-seeking behaviors. The prediction is also consistent with evidence in non-human animals demonstrating that robust, decisive victory experiences interact with post-victory testosterone increases to influence subsequent aggressive behavior (Gleason et al. 2009; Fuxjager et al. 2011; Oliveira 2009; Oliveira et al. 2009; Trainor et al. 2004). To test the moderating role of victory context on the association between testosterone change and competitive decisionmaking, the present study randomly assigned undergraduate men to experience a close or decisive victory against a trained actor (a male confederate posing as another student), measured testosterone changes from before to after the competition, and then asked participants to choose whether they wanted to compete against the same opponent, compete against a new opponent, or complete a non-competitive task. We hypothesized that testosterone change would be positively related to competitive behavior, but only after a decisive victory.

The present study also explored novel psychological mechanisms for post-victory testosterone changes. As reviewed above there is substantial variability in testosterone responses to competition, but the psychological mechanisms remain unclear. Non- 
human animal studies indicate that the effect of testosterone release after winning on future aggressive behavior is driven by increased androgen receptor expression in neural regions implicated in reward processing (e.g., ventral striatum/nucleus accumbens, Fuxjager et al. 2010). Thus, we tested whether post-victory testosterone change would be related to one particular psychological measure implicated in reward: subjective enjoyment of the competitive task, a potential psychological correlate of neural reward activity (e.g., Cikara et al. 2011). We also tested associations between testosterone change and two other psychological factors - perceived likelihood of winning a second competition and affect, but we did not formulate specific hypotheses for these additional measures either because of prior null effects (affect, Mehta and Josephs 2006) or because of a lack of theory or research (perceived likelihood of winning a second competition). Finally, the present research also measured changes in cortisola hormone released by the adrenal glands as part of the hypothalamic-pituitaryadrenal axis response to physical and psychological stress (Dickerson and Kemeny 2004) - to confirm that hormonal effects on competitive behavior were specific to the gonadal axis, in line with prior research on hormone changes in competition (Mehta and Josephs 2006).

\section{Methods}

Participants

Seventy-one male undergraduate students from the University of Oregon enrolled in introductory psychology courses participated in the study in exchange for course credit. All procedures were approved by the University of Oregon Institutional Review Board. The main analyses included 62 participants (details discussed below). The average age of participants used in the analyses was 19.74 years $(S D=1.63)$. The self-reported ethnicity of participants was $73.4 \%$ European-American, $10.9 \%$ Asian or Pacific Islander, $7.8 \%$ Hispanic/Latino, and $7.8 \%$ other.

\section{Procedure}

Experimental sessions were conducted between 11:00 AM and 5:00 PM to control for diurnal rhythms in hormone concentrations (Touitou and Haus 2000). Participants were asked to refrain from eating, drinking, smoking, and brushing their teeth at least $1 \mathrm{~h}$ prior to arriving at their scheduled lab session. Participants provided informed consent prior to participation. Before arriving for the study, participants completed online personality questionnaires, including the Big Five Inventory (John and Srivastava 1999) and the Personality Research Form - Dominance Scale (Jackson 1967). Upon arrival for the experiment, participants were brought into an individual testing room and completed questionnaires relevant to demographics and hormone measurement (Schultheiss and Stanton 2009), the State-Trait Anxiety Inventory (Spielberger 1983), and the Positive and Negative Affect Schedule (Watson, Clark, and Tellegen 1988). Participants then provided a $2 \mathrm{~mL}$ saliva sample, which was immediately stored in a freezer to preserve its contents for analysis through immunoassay procedures (Schultheiss and Stanton 2009). 
Competitive Task The participant and a male confederate acting in the role of a second participant were brought into a larger room and sat at desks facing opposite directions. A timer was placed on each desk. Participants were then asked to read through instructions for the competitive task, the Number Tracking Task (NTT: Schultheiss and Rohde 2002; Mehta and Josephs 2006). In order to increase the status implications of the task, participants were told that the NTT is a measure of spatial intelligence and that the winner would be entered into a random drawing for a $\$ 50$ gift certificate to the University of Oregon Duck Store. This task has been used successfully in past studies on testosterone and competition; participants seem to care about their performance on this task, and participants show robust emotional responses to victory and defeat in the NTT (Mehta and Josephs 2006) similar to patterns observed in other laboratory-based cognitive contests (Gladue, Boechler, and McCaul 1989; Schultheiss et al. 2005).

The NTT consists of a series of puzzles containing a grid of numbers. The goal is to trace through the numbers in sequential order until a highlighted number is reached. The participant and the confederate competed on the same puzzles at the same time. Whoever completed each round in the least amount of time was declared the winner for that round. The participant and confederate competed on six rounds of the task. In each round of the competition, the experimenter placed a puzzle face down on the participant's and the confederate's desks. Participants were instructed to start the timers, begin the puzzle, and say "done" and stop their timers once they had completed the puzzle. The experimenter then announced the winner of the round, recorded completion times, collected the completed puzzle, and placed the next puzzle on the desk. This process was repeated until all six rounds were complete.

The confederate was trained to either barely or decisively lose in each round of the competition. The participant and the confederate competed on each puzzle simultaneously. In the close victory condition, the confederate was trained to lose all six rounds by announcing "done" 1 to $3 \mathrm{~s}$ after the real participant had announced "done". In the decisive victory condition, the confederate was trained to lose all six rounds by announcing "done" 6 to $10 \mathrm{~s}$ after the real participant had announced "done." Because the participant and the confederate competed at the same time on the puzzles, it was necessary for the confederate to lose in each round of competition (the actual participant won in each round). It was not possible for the confederate to win by a fixed margin, as this would have required knowing how long the participant would take to complete the puzzle in order to announce "done" a pre-determined amount of time before the participant finished. We chose this manipulation strategy after piloting the task. Our pilot sessions suggested that having participants compete on the task at the same time increased the competitive nature of the task and that a larger than 6-10 s distance in performance on the task increased suspicion associated with the experimental manipulation. Although results suggest that our experimental manipulation was effective in creating perceptions of a relatively close versus decisive competition, in the Discussion section we address the need for future studies that use stronger experimental manipulations of close versus decisive competition.

Post-Competition Phase After the competition was complete, participants returned to their individual rooms, where they answered the State-Trait Anxiety Inventory and the Positive and Negative Affect Schedule questionnaires again to determine affective changes in response to the competition. All participants also completed an attribution 
questionnaire and a filler task (a word search) (Mehta and Josephs 2006). Fifteen minutes after the end of the competition, a second saliva sample was taken (Mehta and Josephs 2006). This 15-minute delay was chosen because prior research suggests that it takes a few minutes for hormone concentrations in blood to reach saliva (Riad-Fahmy et al. 1987) and because previous research has found that competition outcome influences salivary testosterone changes 15 min after competition, but not immediately after or 30 min after competition (Schultheiss et al. 2005).

Following the second saliva sample, participants completed a choice questionnaire in which they were asked to choose the next experimental task. This questionnaire was modeled closely off of Mehta and Josephs (2006) but was modified to include a third option. Participants chose whether to (a) compete against the same person on six new puzzles of the NTT, (b) compete against a new person on six new puzzles of the NTT, or (c) complete a questionnaire on food, music, and movie preferences that would take about as long to complete as the NTT. After making their choice, participants estimated on a one to seven scale their likelihood of winning if they were to compete on the NTT again $(1=$ Very Unlikely, $7=$ Very Likely). The questionnaire indicated that participants should answer the item even if they had not chosen to compete again. Participants also assessed how much they enjoyed the NTT on a one to seven scale $(1=$ Disliked it a lot, $7=$ Liked it a lot). Participants then filled out open-ended questions designed to assess suspicion associated with the experimental manipulation, after which they were debriefed and dismissed from the experiment.

Approximately half way through data collection we decided to add additional questions about mood states during the competition that are more directly linked to close versus decisive competitions. Participants were asked how fun, competitive, fast, boring, frustrating, stressful, and interesting they perceived the NTT competition to be on 5-point scales. These were included at the very end of the experiment after the suspicion check items to keep the timing and order of all prior measures the same.

\section{Hormone Assays}

Hormone assays were conducted in house at our Social Psychoneuroendocrinology Laboratory within the Sacred Heart Medical Center at the University of Oregon. Seventy-one participants provided saliva samples via passive drool. One participant provided viscous samples, and another participant's samples were contaminated with blood. These two participants were removed from all analyses. Once collected, samples were stored at $-80{ }^{\circ} \mathrm{C}$. After data collection was complete for all participants, the saliva samples were thawed to room temperature, vortexed, centrifuged (10 min@ $3500 \mathrm{rpm}$ ), and aliquoted into individual polypropylene containers. Samples were then refrozen at $-80{ }^{\circ} \mathrm{C}$. Individual aliquots were thawed, vortexed, and centrifuged a second time prior to assaying. This processing protocol (e.g., aliquots that undergo the same number of freeze-thaw cycles prior to assaying) ensures consistent treatment of all samples and is in line with published recommendations (Granger et al. 2004).

Cortisol All saliva samples were analyzed for salivary cortisol using commercially available enzyme immunoassays kits (RE52611, IBL International, Hamburg, Germany). All samples were assayed in duplicate. Intra-assay coefficients of variation 
(CVs) averaged $10.54 \%$. The inter-assay CV averaged across low and high controls was $14.50 \%$. Six participants had samples with elevated intra-assay CVs (>30\%), likely due to inconsistent pipetting technique. These participants were removed from all data analyses involving cortisol, resulting in 63 participants with complete cortisol data. Baseline cortisol means $(M=0.453 \mu \mathrm{g} / \mathrm{dL}, S D=0.364 \mu \mathrm{g} / \mathrm{dL})$ were similar to those reported in past research (e.g., Mehta and Josephs 2006).

Testosterone Saliva samples were assayed for salivary testosterone using commercially available enzyme immunoassays kits (RE52631, IBL International). All samples were assayed in duplicate. Intra-assay CVs averaged $8.46 \%$, and the inter-assay CV averaged across low and high controls was $14.89 \%$. Data from four participants resulted in testosterone concentrations higher than the highest standard for the standard curve for the assay. Additionally, one participant was removed from analyses because hormone data were assayed for only one of the two saliva samples that the participant provided due to a clerical error. These five participants were not included in any data analyses involving IBL testosterone data, resulting in 64 participants with complete IBL testosterone data (IBL Testosterone: $M=262.83 \mathrm{pg} / \mathrm{mL}, S D=166.18 \mathrm{pg} / \mathrm{mL}$ ).

Because a few samples resulted in out of range values in the IBL testosterone assays, we decided to re-test all samples for which there was sufficient volume with Salimetrics testosterone kits to confirm the robustness of the effects in the present study, and to determine whether the effects generalize across assay manufacturers. Twelve participants had insufficient sample volume for these assays, which resulted in 57 total participants with complete Salimetrics testosterone data. Intra-assay CVs for Salimetrics testosterone averaged $5.60 \%$, while the inter-assay CV averaged across low and high controls was $9.02 \%$. (Salimetrics T: $M=118.93 \mathrm{pg} / \mathrm{mL}, S D=33.24 \mathrm{pg} / \mathrm{mL}$ ). There were modest correlations between IBL and Salimetrics testosterone concentration scores (time 1: $r=.32$; time 2: $r=.42$; time two minus time one: $r=.51$, p's $<.05$ ). These results for IBL and Salimetrics testosterone are generally consistent with results reported in quality control reports (quality control reports from IBL can be requested here: http://www.ibl-international.com/en_us/qas-for-salivary-steroids). The primary results in the present study were robust across both IBL and Salimetrics testosterone concentration data as reported in the results section.

\section{Suspicion Check}

Four participants were highly suspicious of the experimental manipulation and were removed, resulting in 62 participants included in the main analyses.

\section{Results}

Initial Analyses

We first conducted analyses to verify that there were no differences in pre-competition psychological or hormone concentrations as a function of our victory type manipulation. As expected, independent samples $t$-tests revealed that there were non-significant 
differences in pre-competition testosterone $(\mathrm{t}(60)=-.01, p=.996)$, pre-competition cortisol $(\mathrm{t}(59)=-1.15, p=.26)$, pre-competition positive affect $(\mathrm{t}(60)=-.23, p=.82)$, and pre-competition negative affect $(\mathrm{t}(60)=-.15, p=.88)$ between the close and decisive victory conditions (see Table 1 for means and standard deviations).tgroup

\section{Effects of Close Versus Decisive Victory on Psychological and Neuroendocrine Changes}

We next conducted repeated measures ANOVAs to test the extent to which the victory experience induced changes in positive affect, negative affect, cortisol change, or testosterone change from before to after the competition. In each of these analyses we entered pre-competition (time 1) and post-competition (time 2) scores as a repeated measures factor and the close versus decisive victory manipulation as a betweensubjects factor. These analyses revealed main effects of time for positive affect $(\mathrm{F}(1,59)=23.70, p<.001)$ and negative affect $(\mathrm{F}(1,59)=12.15, p=.001)$ but nonsignificant Time $x$ Victory Type (close versus decisive) interactions (p's>.10) (see Table 1 for means and standard deviations). These results indicate that participants increased in positive affect and decreased in negative affect after experiencing victory regardless of whether the victory was close or decisive. There were non-significant Time and Time x Victory Type interactions for testosterone and cortisol (p's>.10) (see Table 1). These findings are consistent with prior research on NTT competitions and other cognitive competitions, which also found that victory in the task led to increased positive affect but had no influence on mean testosterone or cortisol changes (Mehta and Josephs 2006; Schultheiss et al. 2005). Another study of cognitive competition in

Table 1 Descriptive statistics for hormone and psychological measures

\begin{tabular}{|c|c|c|c|c|c|c|}
\hline & \multicolumn{2}{|c|}{$\begin{array}{l}\text { Full sample } \\
(n=62)\end{array}$} & \multicolumn{2}{|c|}{$\begin{array}{l}\text { Close victory } \\
(n=30)\end{array}$} & \multicolumn{2}{|c|}{$\begin{array}{l}\text { Decisive } \\
\text { victory }(n=32)\end{array}$} \\
\hline & M & SD & M & SD & M & $\mathrm{SD}$ \\
\hline IBL Pre-competition Testosterone (pg/mL) & 265.1 & 163.4 & 262.9 & 162.3 & 267.3 & 167.0 \\
\hline IBL Post-competition Testosterone (pg/mL) & 261.1 & 164.2 & 279.8 & 169.2 & 243.6 & 160.1 \\
\hline Pre-competition cortisol $(\mu \mathrm{g} / \mathrm{dL})^{\mathrm{a}}$ & .45 & .33 & .48 & .31 & .42 & .34 \\
\hline Post-competition cortisol ( $\mu \mathrm{g} / \mathrm{dL})$ & .42 & .23 & .43 & .23 & .41 & .24 \\
\hline Pre-competition positive affect ${ }^{b}$ & 2.80 & .72 & 2.82 & .83 & 2.79 & .61 \\
\hline Post-Competition Positive affect & 3.17 & .83 & 3.22 & .96 & 3.13 & .70 \\
\hline Pre-competition negative affect ${ }^{c}$ & 1.50 & .52 & 1.51 & .54 & 1.49 & .52 \\
\hline Post-competition negative affect & 1.36 & .44 & 1.40 & .46 & 1.32 & .43 \\
\hline Enjoyment of number tracking task ${ }^{\mathrm{d}}$ & 5.44 & .87 & 5.59 & .73 & 5.31 & .97 \\
\hline Perceived likelihood of winning a second competition ${ }^{\mathrm{e}}$ & 5.15 & .98 & 5.17 & .85 & 5.13 & 1.10 \\
\hline
\end{tabular}

${ }^{\text {a }}$ Full sample size for cortisol measures was $n=61$. Means and standard deviations reported here were calculated from the untransformed pre-competition cortisol distribution. ${ }^{b}$ Positive Affect subscale of PANAS (Watson, Clark, and Tellegen 1988); scores can range from one to five. ${ }^{\mathrm{c}}$ Negative Affect subscale of PANAS (Watson, Clark, and Tellegen 1988); scores can range from one to five. ${ }^{\mathrm{d}}$ Rated on a 7-point scale $(1=$ Disliked a lot, $7=$ Liked it a lot $)(n=61$ for this measure $){ }^{\text {e }}$ Rated on a 7 -point scale $(1=$ Very Unlikely, 7 = Very Likely) ( $n=61$ for this measure) 
males also revealed non-significant differences between a close versus decisive victory in mean testosterone or cortisol change (Gladue et al. 1989).

We next tested whether the experimental manipulation influenced self-reports of how fun, competitive, fast, boring, frustrating, stressful, and interesting participants perceived the Number Tracking Task competition to be. As reported in the methods section, we added these additional questions approximately half way through study execution and thus the sample size for these analyses was reduced to 31 participants. A multivariate ANOVA analysis revealed a statistically significant multivariate effect of the experimental manipulation on these dependent measures $(\mathrm{F}(7,23)=2.60, p=.04)$. Follow-up univariate tests revealed that participants in the close victory condition perceived the competition to be more competitive $(M=4.50, S E=.22)$ and fast $(M=4.29, S E=.17)$ than participants in the decisive victory condition (competitive: $M=3.88, S E=.20$; fast: $M=3.82, S E=.15$ ) (competitive: $\mathrm{F}(1,29)=4.41, p=.045$; fast: $\mathrm{F}(1,29)=4.20, p=.05)$. These results suggest that our experimental manipulation was effective in influencing perceptions consistent with a close versus decisive competition. Participants in the close victory condition also reported that the task was marginally more fun $(M=4.07, S E=.18)$ than participants in the decisive victory condition $(M=3.55, S E=.16)(\mathrm{F}(1,29)=3.13, p=.087)$. All other univariate effects were non-significant ( $\mathrm{p}$ 's $>.18)$.

\section{Competitive Decision-Making}

Across the entire sample, 32 out of the 62 participants (51.6\%) chose to complete the entertainment questionnaire, 26 out of the 62 participants $(41.9 \%)$ chose to compete against a new opponent, but only 4 out of 62 participants $(6.5 \%)$ chose to compete against the same opponent. Given the small number of participants who chose to compete against the same opponent, analyses examining predictors of competitive decision-making were conducted in two ways. First, we combined participants who chose to compete against the same opponent with participants who to chose to compete against a new opponent in order to compare our results to prior research that gave participants a choice between the competitive and non-competitive task only (Mehta and Josephs 2006). Next, we removed the four participants who chose to compete against the same opponent to confirm that effects were driven by the decision to compete against a new opponent compared to choosing the noncompetitive task. We did not conduct formal statistical analyses for participants who chose to compete against the same opponent because of the low frequency at which this option was chosen.

Testosterone Change We tested the hypothesis that testosterone change and victory type would interact to predict competitive decision-making. Specifically, we expected that testosterone change would positively relate to the propensity to compete only after a decisive victory. We conducted a binary logistic regression analysis in which we entered Victory Type $(0=$ decisive victory, $1=$ close victory $)$ in Step 1 , Testosterone Change (centered) in Step 2, and the Victory Type x Testosterone Change interaction in Step 3 as predictors of the decision to compete $(0=$ complete non-competitive task, 1 = compete again collapsed across decisions to compete against a new or the same opponent). This analysis revealed a main effect of Victory Type in Step 1 
$\left(\chi^{2}(1)=7.95, p=.005\right)$ and a non-significant main effect of Testosterone Change in Step $2\left(\chi^{2}(1)=.85, p=.36\right)$. In support of the hypothesis that victory type moderates the association between testosterone change and competitive decision-making, there was a statistically significant Victory Type $\mathrm{x}$ Testosterone Change interaction in Step $3\left(\chi^{2}(1)=3.95, p=.047\right)$.

To interpret the Victory Type $\mathrm{x}$ Testosterone Change interaction we used the logistic regression intercept and slope estimates to plot the interaction (http://www. jeremydawson.co.uk/slopes.htm) and conducted separate binary logistic analyses in the close and decisive victory conditions (see Fig. 1a) (Aiken and West 1991). Consistent with our hypothesis, testosterone change was positively related to choosing
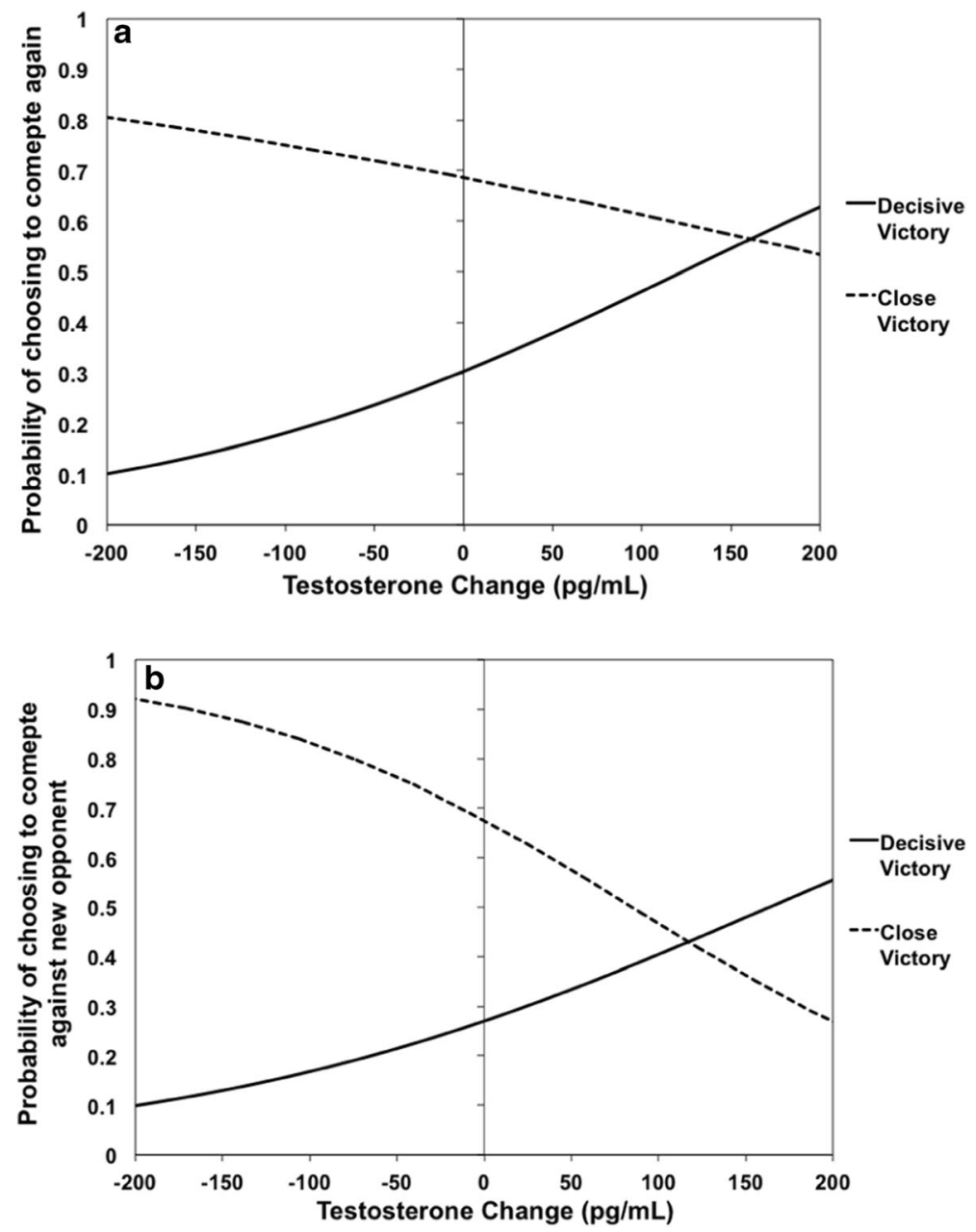

Fig. 1 a Victory Type $x$ Testosterone Change interaction on the likelihood of choosing to compete again (versus choosing the alternative non-competitive task). Decisions to compete against the same opponent and decisions to compete against a new opponent were combined for this analysis. b Victory Type $\mathrm{x}$ Testosterone Change interaction on the likelihood of choosing to compete against a new opponent (versus choosing the alternative non-competitive task). The four participants who chose to compete against the same opponent were removed for this analysis 
to compete in the decisive victory condition $\left(B=.92, S E=.57, e^{B}=2.51, \chi^{2}(1)=3.98\right.$, $p=.046)$ (Fig. 1a, solid line) but not in the close victory condition $(B=-.44, S E=.50$, $e^{B}=.65, \chi^{2}(1)=.82, p=.36$ ) (Fig. 1a, striped line). We also calculated mean testosterone change as a function of victory type and competitive choice. Consistent with the positively-sloping solid line in Fig. 1a, individuals in the decisive victory condition who chose to compete increased in testosterone $(n=10$, $M=46.85, S E=46.56$ ), whereas individuals in the decisive victory condition who chose to complete the alternative non-competitive task decreased in testosterone $(n=22$, $M=-55.68, S E=31.39$ ). Consistent with slightly negatively-sloping striped line in Fig. $1 \mathrm{a}$, individuals in the close victory condition who chose to compete again showed little change in testosterone $(n=20, M=3.88, S E=25.72)$, whereas individuals in the close victory condition who chose to complete the alternative non-competitive task showed a testosterone increase $(n=10, M=43.09, S E=36.37)$.

Next we removed the four participants who chose to compete against the same opponent from the analysis to confirm that the same interaction effect would remain. Consistent with the prior analysis, there was a Victory Type $\mathrm{x}$ Testosterone Change interaction in Step $3\left(\chi^{2}(1)=5.54, p=.019\right)$ (Fig. 1b). The pattern of the interaction shows a positive slope between testosterone change and the decision to compete against a new opponent in the decisive victory condition $\left(B=.83, S E=.64, e^{B}=2.28\right)$ (Fig. 1b, solid line), but a negative slope between testosterone change and the decision to compete against a new opponent in the close victory condition $(B=-1.18, S E=.77$, $\left.e^{B}=0.31\right)$ (Fig. 1b, striped line). The statistically significant interaction term indicates that these slopes were statistically different from each other. We also calculated mean testosterone change as a function of victory type and competitive choice. Consistent with the positively-sloping solid line in Fig. 1b, individuals in the decisive victory condition who chose to compete against a new opponent increased in testosterone $(n=8, M=26.25, S E=50.92)$, whereas individuals in the decisive victory condition who chose to complete the alternative noncompetitive task decreased in testosterone ( $n=22, M=-55.68, S E=30.70)$. Consistent with the negatively-sloping striped line in Fig. 1b, individuals in the close victory condition who chose to compete against a new opponent decreased in testosterone $(n=18, M=-20.31, S E=22.63)$, whereas individuals in the close victory condition who chose to complete the alternative non-competitive task increased in testosterone $(n=10, M=43.09, S E=30.37)$.

The above analyses used post-competition minus pre-competition testosterone change scores, but there was a similar Victory Type $\mathrm{x}$ Testosterone Change interaction on the decision to compete against a new opponent compared to choosing the noncompetitive task when using percent change in testosterone $\left(\chi^{2}(1)=3.49, p=.062\right)$ and residualized testosterone change $\left(\chi^{2}(1)=6.45, p=.011\right)$ (residualized testosterone change scores were calculated by saving the unstandardized residuals from a regression analysis with pre-competition testosterone as a predictor and postcompetition testosterone as the dependent variable, see Mehta and Josephs 2006; Schultheiss et al. 2005).

Pre-Competition Testosterone, Pre-Competition Cortisol, and Cortisol Change Next we conducted binary logistic regression analyses to test whether pre-competition testosterone or pre-competition cortisol (log-transformed) were related to competitive 
decision-making or interacted with victory type to predict competitive decision-making. These analyses revealed null effects ( $p$ 's $>.30$ ). Additional binary logistic analyses revealed non-significant effects of Cortisol Change and Victory Type $\mathrm{x}$ Cortisol Change interactions on competitive decisions ( $p$ 's $>.20$ ). Overall, these analyses indicate that testosterone change interacted with victory type to predict competitive decisions, but pre-competition testosterone, pre-competition cortisol, and cortisol change failed to predict competitive decisions.

\section{Enjoyment of the Competitive Task}

We next tested whether testosterone change interacted with victory type to predict how much participants reported that they had enjoyed the NTT competition. A multipleregression analysis with Victory Type, Testosterone Change (IBL time two minus time 1 scores, centered), and the Victory Type $\mathrm{x}$ Testosterone Change interaction as predictors of task enjoyment revealed a statistically significant Victory Type $\mathrm{x}$ Testosterone Change interaction $(\beta=-.40, \mathrm{t}(57)=-2.82, p=.007)$. As shown in Fig. 2, testosterone change was positively related to enjoyment of the competitive task in the decisive victory condition (Fig. 2, solid line, $B=.41, S E=.13, \mathrm{t}(57)=3.21, p=.002$ ), but testosterone change was non-significantly negatively related to enjoyment of the competitive task in the close victory condition (Fig. 2, striped line, $B=-.28, S E=.21$, $\mathrm{t}(57)=-1.34, p=.19)$. The Victory Type $\mathrm{x}$ Testosterone Change interaction on enjoyment of the competitive task also emerged when using IBL percent testosterone change scores $(\beta=-.39, \mathrm{t}(57)=-2.43, p=.018)$ and IBL residualized testosterone change scores $(\beta=-.41, \mathrm{t}(57)=-2.68, p=.010)$.

There were non-significant effects of cortisol change and victory type $\mathrm{x}$ cortisol change interactions on enjoyment of the competitive task ( $p$ 's $>.10)$. There were also non-significant main effects and interaction effects for pre-competition testosterone and cortisol ( $p$ 's $>.10)$. Overall, these analyses indicate that testosterone change interacted

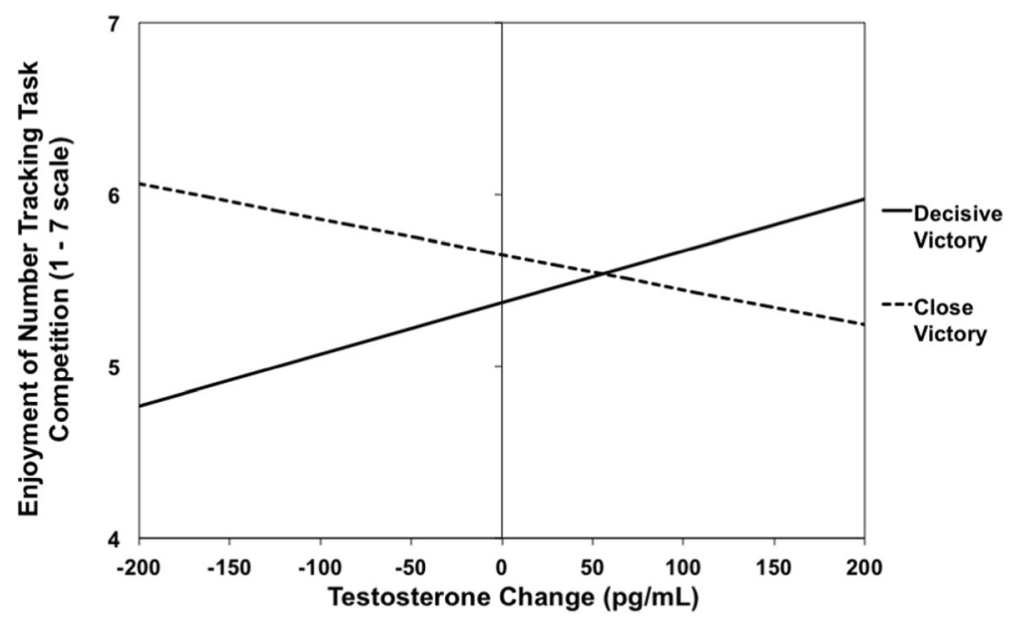

Fig. 2 Victory Type $x$ Testosterone Change interaction on self-reported enjoyment of the competitive task $(1=$ Disliked it a lot, $7=$ Liked it a lot $)$. The minimum enjoyment score was 4 and the maximum was 7 
with victory type to predict self-reported enjoyment of the competitive task, but precompetition testosterone, pre-competition cortisol, and cortisol change failed to predict task enjoyment ${ }^{1}$.

\section{Mediation Analyses}

The analyses reported above indicate that testosterone change interacted with victory type to predict both enjoyment of the competitive task and the decision to compete again. Moreover, binary logistic regression analyses revealed that enjoyment of the competitive task was a statistically significant predictor of the decision to compete again (both when including participants who chose to compete against the same opponent and when excluding them, p's<.05). Thus, we conducted exploratory bootstrapping analyses to test whether enjoyment of the competitive task statistically mediated the victory type $\mathrm{x}$ testosterone change interaction on competitive decision-making (Preacher and Hayes 2004). However, these analyses failed to provide robust evidence for statistical mediation (confidence intervals for indirect effects overlapped with zero $)^{2}$.

\section{Estimates of Likelihood of Winning}

We used multiple regression analyses to examine whether testosterone or cortisol were related to estimates of the likelihood of winning a second Number Tracking Task competition. There were non-significant main effects or interactions with victory type for pre-competition testosterone, pre-competition cortisol, testosterone change, and cortisol change in these analyses ( $p$ 's $>.06$ ). Further, estimates of the likelihood of winning were unrelated to the decision to compete again ( $p$ 's $>.20)$.

\footnotetext{
${ }^{1}$ Our main results replicated when using Salimetrics kits to measure testosterone concentrations. There were Victory Type $\mathrm{x}$ Testosterone Change interactions on the decision to compete against a new opponent compared to choosing the non-competitive task when using Salimetrics post-competition minus pre-competition testosterone scores $\left(\chi^{2}(1)=4.96, p=.026\right)$, Salimetrics percent testosterone change scores $\left(\chi^{2}(1)=4.95, p=.026\right)$, and Salimetrics residualized testosterone change scores $\left(\chi^{2}(1)=6.76, p=.009\right)$. There were Victory Type $\mathrm{x}$ Testosterone Change interactions on task enjoyment when using Salimetrics time two minus time one scores $(\beta=-.44, \mathrm{t}(49)=-2.49, p=.016)$, Salimetrics percent testosterone change scores $(\beta=-.45, \mathrm{t}(49)=-2.40$, $p=.020)$, and Salimetrics residualized testosterone change scores $(\beta=-.43, \mathrm{t}(49)=-2.38, p=.020)$. These analyses indicate that the Victory Type $\mathrm{x}$ Testosterone Change interactions on task enjoyment and competitive decision-making were remarkably robust across assay manufacturers and multiple ways of calculating testosterone change.

${ }^{2}$ In line with recent recommendations for increasing transparency in published research (e.g., Simmons et al. 2011), we conducted additional analyses to determine how decisions to include or exclude certain data points impacted the overall results. When we included the four highly suspicious participants in our analyses, the Victory Type $\mathrm{x}$ Testosterone Change interaction on the decision to compete against a new opponent remained, as did the Victory Type $\mathrm{x}$ Testosterone Change interaction on enjoyment of the competitive task (with both IBL and Salimetrics residualized T change scores, p's $<.05$ ). We also went back to our open-ended suspicion check data and applied more stringent criteria for excluding participants, which resulted in an additional nine participants who could be categorized as at least somewhat suspicious. Despite the large reduction in statistical power associated with removing these nine participants, there were still Victory Type $\mathrm{x}$ Testosterone Change interactions on competitive decision-making and enjoyment of the competitive task that were consistent with our main analyses (IBL and Salimetrics residualized T change scores, p-values ranging between .023 and .076). These analyses demonstrate the robustness of the effects across different inclusion/exclusion criteria.
} 


\section{Hormones and Affect}

We also examined associations between hormones and affective measures. We did not expect statistically significant effects given a lack of overlap between self-reported affect and hormones in prior research on NTT or other cognitive competitions (Mehta and Josephs 2006; Schultheiss et al. 2005). These analyses revealed that precompetition cortisol was positively related to post-competition positive affect $(r=.32$, $p=.017)$ and change in positive affect from pre- to post-competition $(r=.29, p=.034)$. There were non-significant correlations for cortisol change, and there were no robust correlations that replicated across multiple measures for pre-competition testosterone or testosterone change.

\section{Discussion}

Theories of testosterone dynamics and status propose that post-victory testosterone increases should stimulate the desire to enter into future competition, but the only prior study that examined this association reported a null result (Mehta and Josephs 2006). The present study extended this work by showing that a post-victory testosterone increase does predict a greater propensity to compete, but only after a decisive victory. Testosterone change showed the opposite association with competitive decisions after a close victory. These effects were driven by the decision to compete against a new opponent. In fact, only four participants $(6.5 \%$ of the sample) chose to compete against the same opponent. The effects were specific to the gonadal axis, as similar effects did not emerge for cortisol. Taken together, our findings advance theory on testosterone and status in suggesting that a testosterone increase predicts future attempts at gaining status (entering a second competition) only after a clear, stable rise in status (decisive victory) but not after an uncertain, unstable rise in status (close victory).

In addition to advancing knowledge on contextual moderators of testosterone change-behavior associations, the present study also revealed a novel psychological correlate of post-victory testosterone change: self-reported enjoyment of the competitive task. Testosterone change was positively correlated with task enjoyment after a decisive victory. Although we are the first to report this association (at least to our knowledge), this finding converges with theories linking testosterone to reward processing and reinforcement. Previous research has found that rats prefer places that were previously paired with testosterone injections, and follow-up studies indicate that this effect of testosterone on place preference is mediated by testosterone's interactions with dopamine in the ventral striatum, a brain area associated with reward (Packard et al. 1997, 1998). Additional research in California mice suggests that post-victory testosterone increases influence future aggressive behavior via increased androgen receptor expression in the ventral striatum (Fuxjager et al. 2010), likely through testosterone's interactions with dopamine. Consistent with these non-human animal studies, exogenous testosterone administration in humans augments ventral striatum activity to financial reward cues (Hermans et al. 2010; Op de Macks et al. 2011), and ventral striatum activity positively correlates with subjective pleasure responses to successful performance in competition (e.g., watching one's team play favorably) as well as aggressive motivation towards opponents (Cikara et al. 2011). This cross-species 
evidence converges to suggest that a dynamic testosterone increase after a decisive victory may enhance future competitive behavior and subjective task enjoyment through testosterone's effects on reward regions such as the ventral striatum (but other possible neural mechanisms include changes in amygdala, prefrontal cortex, or amygdala-prefrontal cortex connectivity, Peper et al. 2011).

Although our study revealed a positive association between testosterone change and decisions to compete in the decisive victory condition, there was a surprising negative association between testosterone change and the propensity to compete against a new opponent in the close victory condition that was accompanied by a similar pattern for task enjoyment (striped lines in Figs. 1b and 2). The BMS proposes that post-victory testosterone increases should stimulate future attempts at gaining or maintaining high status (Mazur 1985; Mazur and Booth 1998), but little is known about the conditions under which testosterone increases encourage status-gaining versus status-maintaining behaviors. Although speculative, one interpretation of our results is that a testosterone increase after a decisive victory may encourage further attempts to gain status (competing again), whereas a testosterone increase after a close victory may encourage attempts to maintain one's higher status position (avoiding further competition). Close victories model unstable hierarchies in which the winner's higher status position is uncertain, and this uncertainty coupled with a drive toward status (testosterone increase) may lead to a status-maintaining behavioral strategy. After all, actively seeking out further dominance confrontations is risky given that one's higher status position was only barely earned; a second competition could possibly lead to a loss of status rather than a status gain. Consistent with this argument is evidence that unstable and illegitimate high-status positions lead to risk aversion and behavioral avoidance, whereas stable and legitimate high-status positions lead to risk taking and behavioral approach (Jordan et al. 2011; Lammers et al. 2008). Importantly, post-victory testosterone changes in the present study were not associated with one's perceived likelihood of winning a second competition, suggesting that testosterone change does not operate on status-seeking or maintenance through conscious perceptions of winning ability. Instead, we found preliminary evidence that testosterone increases after a close victory may be associated with reduced reward processing (reduced enjoyment of the competitive task, striped line in Fig. 2), which complements the argument detailed above that testosterone increases after a decisive victory may be associated with increased reward processing. The present study is the first to demonstrate a moderating role of close versus decisive victory on the behavioral and psychological consequences of testosterone change, but future research is needed to confirm the robustness of these effects and provide additional tests of the underlying mechanisms.

The current research builds upon a growing literature in humans suggesting that short-term testosterone changes both within and outside of competition dynamically tunes a range of behaviors that may have adaptive benefits for survival and reproduction, including competitive behavior and performance (Carré and McCormick 2008; Mehta and Josephs 2006; Zilioli and Watson 2014), aggression (Carré et al. 2009, 2010, 2011, 2013; Geniole et al. 2011), mating behavior (Roney et al. 2007; van der Meij et al. 2011), trust perceptions (Carré et al. 2014), social and financial risk-taking (Apicella et al. 2014; Ronay and von Hippel 2010), and learning (Schultheiss et al. 2005). The effect sizes we observed in the present study (e.g., standardized beta of .40 for close/decisive victory $\mathrm{x}$ testosterone change interaction on task enjoyment) are in 
line with effect sizes observed in this prior research (e.g., $r=.34$ for association between testosterone change and aggressive behavior, Geniole et al. 2011). Although some of these studies tested whether a rise versus drop in status (victory versus defeat) moderates the association between post-competition testosterone change and behavioral outcome measures, researchers have generally failed to consider the potential moderating role of hierarchical stability versus instability (close versus decisive victory) (see also Zilioli and Watson 2014; Zilioli et al. 2014). The present findings suggest that greater focus should be placed on studying hierarchy stability within this research tradition. More broadly, our findings highlight the need to expand the search for contextual moderators in order to rapidly advance knowledge on testosterone dynamics and status in humans.

There are some limitations and open questions that should be addressed in future studies. We used a laboratory cognitive competition (the NTT) because it allows for experimental control in the victory experience and because participants show robust affective changes to NTT competitions (Mehta and Josephs 2006) similar to other cognitive competitions (Schultheiss et al. 2005). Nevertheless, it is possible that participants in the present study did not perceive the NTT as important or did not care whether they won or lost. This possibility seems unlikely because the victory experience produced a robust increase in positive affect and decrease in negative affect similar to prior research, but future studies should include questions related to perceived importance of the competition in order test its associations or interactions with competition-related testosterone dynamics.

The present study used a subtle manipulation of relatively close versus decisive victory in which participants won six puzzles in a row by either 1-3 s per round (close victory) or 6-10 s per round (decisive victory). This manipulation was effective in influencing perceptions consistent with a close versus decisive competition (perceptions of how competitive and fast the contest was) and competitive decision-making. We chose this experimental manipulation because pilot testing suggested that having the participant compete against the confederate at the same time in each round increases the competitive nature of the contest. However, this manipulation strategy requires that the participant win in every round (confederate loses). It is not possible for the participant to lose by a controlled margin with this approach because doing so would require the confederate to know exactly how long the participant would take to finish the puzzle in order to announce "done" a fixed period of time before the participant finishes. This may have been possible had we used a different approach such as Study 1 of Zilioli et al. 2014 in which the participant completed the puzzle first followed by the confederate, which allows the confederate to adjust completion times to be faster or slower than the participant by a fixed margin. However, this alternative approach has limitations in that it likely decreases the competitive nature of the task because the participant and the confederate are not competing on puzzles at the same time. It will be important for future studies to use stronger manipulations of close versus decisive victory in order to complement the present research. For example, future research can have participants compete on rigged computer-based tasks in which the participant is led to believe they won 4 rounds and lost 2 rounds (close victory) or won all six rounds (decisive victory) (e.g., using rigged video games, Carré et al. 2013; Study 2, Zilioli et al. 2014). Future research can also implement the approach used in Study 1 of Zilioli et al. 2014 in order to create a variety of close and decisive victory experiences in the NTT. 
Victory in the present study did not produce a mean increase in testosterone, but there was substantial variation in testosterone responses that were related to task enjoyment and competitive decision-making. Prior research that used NTT competitions or other cognitive tasks also found non-significant mean testosterone changes after victory with variability in testosterone responses that were associated with psychological factors and behavior (Carré et al. 2009; Mehta and Josephs 2006; Schultheiss et al. 2005; Zilioli et al. 2014). Our results along with these prior studies suggest that individual differences in pre- to post-competition testosterone changes predict future behavior even in the absence of a mean testosterone change, an insight that provides an important theoretical advance to research on testosterone dynamics in competition (see recent reviews, Carré et al. 2011; Oliveira and Oliveira 2014; Salvador and Costa 2009). Nevertheless, it will be important to test whether the present results emerge in competitions that are expected to produce a mean testosterone increase after victory (Carré et al. 2013; Edwards, Wetzel, and Wyner 2006; Gladue et al. 1989; Zilioli and Watson 2014). Similarly, there was a non-significant cortisol change from before to after victory in the current study consistent with prior NTT competitions and other cognitive competitions (Mehta and Josephs 2006; Wirth et al. 2006; Zilioli et al. 2014), but it will be important to test whether the present results extend to other competitive domains that are expected to produce non-significant cortisol changes or a mean cortisol decrease after victory (e.g., Study 1, Mehta et al. 2008; Jiménez et al. 2012; Stanton et al. 2010; Wirth et al. 2006) as well as competitions that are expected to produce a mean cortisol increase after victory (e.g., Edwards et al. 2006).

Our study measured naturally occurring changes in testosterone from before to after an experimentally manipulated close or decisive victory experience. We timed the sequence of events in our study so that testosterone changes were measured prior to competitive decisions and task enjoyment. This design allows us to claim that postvictory testosterone change predicts subsequent competitive behavior, but we did not experimentally manipulate testosterone and thus we cannot be sure that testosterone directly caused task enjoyment or competitive behavior. However, non-human animal studies have demonstrated causality (Gleason et al. 2009; Fuxjager et al. 2011; Oliveira 2009; Oliveira et al. 2009; Trainor et al. 2004). For example, one study of male California mice found that robust victory experiences paired with an injection that produced short-term elevations in testosterone increased future aggressive behavior towards a new opponent (Trainor et al. 2004). Follow-up studies showed similar results in male cichlid fish (Oliveira et al. 2009) and identified neural mechanisms (Fuxjager et al. 2010). These findings suggest that post-victory testosterone changes may also have a causal influence on human social behavior, but human studies in which testosterone concentrations are exogenously altered are required to confirm causality.

The present results are generally consistent with non-human animal studies demonstrating that robust, decisive victory experiences coupled with a testosterone increase stimulates subsequent aggressive behavior (Gleason et al. 2009; Fuxjager et al. 2011; Oliveira 2009; Oliveira et al. 2009; Trainor et al. 2004). At the same time, other nonhuman animal studies demonstrate that testosterone is most strongly related to social behavior in unstable hierarchies (e.g., during periods of social competition such as the mating season) compared to stable hierarchies (when social competition is absent such as the non-mating season) (e.g., lemurs, Cavigelli and Pereira 2000; baboons, Beehner et al. 2006; Sapolsky 1991; birds, Wingfield et al. 1990). However, these non-human 
animal studies tend to operationalize hierarchical stability as periods of social competition (instability) versus no competition (stability) and do not differentiate between close and decisive competitions. Thus, the present results cannot be compared directly to these prior studies. Although much more research needs to be conducted, one possibility is that a testosterone increase during periods of social competition may be more likely to stimulate status-seeking behaviors compared to periods of no competition, whereas a testosterone increase after a decisive victory may be more likely to stimulate status-seeking behaviors compared to a testosterone increase after a close victory. Future studies that examine testosterone-behavior associations across periods of no competition, close competition, and decisive competition are required to test these predictions directly.

The present study examined male competitors, but future research should test whether similar effects emerge in women. Some studies have indeed shown associations between endogenous basal testosterone concentrations and female behavior that converge with results in males (Josephs et al. 2003, 2006; Mehta and Josephs 2010; Mehta et al. 2008, 2009; Newman et al. 2005), and others have shown that testosterone fluctuates in competition similarly across males and females (Jiménez et al. 2012). These studies suggest that the present results may indeed extend to women. However, other research shows null associations between testosterone and aggressive behavior in women (Carré et al. 2011) or shows sex differences in testosterone dynamics (Kivlighan et al. 2005). Thus, a key future direction is to understand the mechanisms (both biological and sociocultural) for these similarities and differences in testosterone function across the sexes.

Acknowledgments We thank Cassandra Brandes, Leo Edmunson, and Adam Tratner for their assistance.

Author Contributions NAS and PHM designed the study; NAS conducted the study with assistance from ELK; NAS, ELK, and PHM analyzed the data; NAS conducted hormone assays, and BL assisted with hormone data interpretation. PHM, NAS, and BL wrote the first draft of the paper, and all authors provided comments on subsequent drafts.

\section{References}

Aiken, L. S., West, S. G. (1991) In Multiple regression: Testing and interpreting interactions, Sage Publications, London.

Apicella, C. L., Dreber, A., \& Mollerstrom, J. (2014). Salivary testosterone change following monetary wins and losses predicts future financial risk-taking. Psychoneuroendocrinology, 39, 58-64.

Archer, J. (2006). Testosterone and human aggression: An evaluation of the challenge hypothesis. Neuroscience \& Biobehavioral Reviews, 30(3), 319-345.

Archer, J., Graham-Kevan, N., \& Davies, M. (2005). Testosterone and aggression: A reanalysis of Book, Starzyk, and Quinsey's (2001) study. Aggression and Violent Behavior, 10(2), 241-261.

Beehner, J. C., Bergman, T. J., Cheney, D. L., Seyfarth, R. M., \& Whitten, P. L. (2006). Testosterone predicts future dominance rank and mating activity among male chacma baboons. Behavioral Ecology and Sociobiology, 59(4), 469-479.

Carré, J. M., \& McCormick, C. M. (2008). Aggressive behavior and change in salivary testosterone concentrations predict willingness to engage in a competitive task. Hormones and Behavior, 54(3), 403-409.

Carré, J. M., Putnam, S. K., \& McCormick, C. M. (2009). Testosterone responses to competition predict future aggressive behaviour at a cost to reward in men. Psychoneuroendocrinology, 34(4), 561-570. 
Carré, J. M., Gilchrist, J. D., Morrissey, M. D., \& McCormick, C. M. (2010). Motivational and situational factors and the relationship between testosterone dynamics and human aggression during competition. Biological Psychology, 84(2), 346-353.

Carré, J. M., McCormick, C. M., \& Hariri, A. R. (2011). The social neuroendocrinology of human aggression. Psychoneuroendocrinology, 36(7), 935-944.

Carré, J. M., Campbell, J. A., Lozoya, E., Goetz, S. M., \& Welker, K. M. (2013). Changes in testosterone mediate the effect of winning on subsequent aggressive behaviour. Psychoneuroendocrinology, 38(10), 2034-2041.

Carré, J. M., Baird-Rowe, C. D., \& Hariri, A. R. (2014). Testosterone responses to competition predict decreased trust ratings of emotionally neutral faces. Psychoneuroendocrinology, 49, 7983.

Cavigelli, S. A., \& Pereira, M. E. (2000). Mating season aggression and fecal testosterone levels in male ringtailed lemurs (Lemur catta). Hormones and Behavior, 37(3), 246-255.

Cikara, M., Botvinick, M. M., \& Fiske, S. T. (2011). Us versus them social identity shapes neural responses to intergroup competition and harm. Psychological Science.

Dickerson, S. S., \& Kemeny, M. E. (2004). Acute stressors and cortisol responses: A theoretical integration and synthesis of laboratory research. Psychological Bulletin, 130(3), 355.

Edwards, D. A., Wetzel, K., \& Wyner, D. R. (2006). Intercollegiate soccer: Saliva cortisol and testosterone are elevated during competition, and testosterone is related to status and social connectedness with teammates. Physiology \& Behavior, 87(1), 135-143.

Fuxjager, M. J., Forbes-Lorman, R. M., Coss, D. J., Auger, C. J., Auger, A. P., \& Marler, C. A. (2010). Winning territorial disputes selectively enhances androgen sensitivity in neural pathways related to motivation and social aggression. Proceedings of the National Academy of Sciences, 107(27), 12393-12398.

Fuxjager, M. J., Oyegbile, T. O., \& Marler, C. A. (2011). Independent and additive contributions of postvictory testosterone and social experience to the development of the winner effect. Endocrinology, 152(9), 3422-3429.

Geniole, S. N., Carré, J. M., \& McCormick, C. M. (2011). State, not trait, neuroendocrine function predicts costly reactive aggression in men after social exclusion and inclusion. Biological Psychology, $87(1), 137-145$.

Gladue, B. A., Boechler, M., \& McCaul, K. D. (1989). Hormonal response to competition in human males. Aggressive Behavior, 15(6), 409-422.

Gleason, E. D., Fuxjager, M. J., Oyegbile, T. O., \& Marler, C. A. (2009). Testosterone release and social context: When it occurs and why. Frontiers in Neuroendocrinology, 30(4), 460-469.

Granger, D. A., Shirtcliff, E. A., Booth, A., Kivlighan, K. T., \& Schwartz, E. B. (2004). The "trouble" with salivary testosterone. Psychoneuroendocrinology, 29(10), 1229-1240.

Hermans, E. J., Bos, P. A., Ossewaarde, L., Ramsey, N. F., Fernández, G., \& Van Honk, J. (2010). Effects of exogenous testosterone on the ventral striatal BOLD response during reward anticipation in healthy women. NeuroImage, 52(1), 277-283.

Jackson, D. (1967). Personality research form manual. New York: Research Psychologists Press.

Jiménez, M., Aguilar, R., \& Alvero-Cruz, J. R. (2012). Effects of victory and defeat on testosterone and cortisol response to competition: Evidence for same response patterns in men and women. Psychoneuroendocrinology, 37(9), 1577-1581.

John, O. P., \& Srivastava, S. (1999). The big five trait taxonomy: History, measurement, and theoretical perspectives. In L. A. Pervin \& O. P. John (Eds.), Handbook of personality: Theory and research (pp. 102-138). New York: Guilford Press.

Jordan, J., Sivanathan, N., \& Galinsky, A. D. (2011). Something to lose and nothing to gain the role of stress in the interactive effect of power and stability on risk taking. Administrative Science Quarterly, 56(4), 530-558.

Josephs, R. A., Newman, M. L., Brown, R. P., \& Beer, J. M. (2003). Status, testosterone, and human intellectual performance stereotype threat as status concern. Psychological Science, 14(2), 158-163.

Josephs, R. A., Sellers, J. G., Newman, M. L., \& Mehta, P. H. (2006). The mismatch effect: When testosterone and status are at odds. Journal of Personality and Social Psychology, 90(6), 999.

Kivlighan, K. T., Granger, D. A., \& Booth, A. (2005). Gender differences in testosterone and cortisol response to competition. Psychoneuroendocrinology, 30(1), 58-71.

Lammers, J., Galinsky, A. D., Gordijn, E. H., \& Otten, S. (2008). Illegitimacy moderates the effects of power on approach. Psychological Science, 19(6), 558-564.

Mazur, A. (1985). A biosocial model of status in face-to-face primate groups. Social Forces, 64(2), 377-402. 
Mazur, A., \& Booth, A. (1998). Testosterone and dominance in men. Behavioral and Brain Sciences, 21(03), 353-363.

Mehta, P. H., \& Josephs, R. A. (2006). Testosterone change after losing predicts the decision to compete again. Hormones and Behavior, 50(5), 684-692.

Mehta, P. H., \& Josephs, R. A. (2010). Testosterone and cortisol jointly regulate dominance: Evidence for a dual-hormone hypothesis. Hormones and Behavior, 58(5), 898-906.

Mehta, P. H., Jones, A. C., \& Josephs, R. A. (2008). The social endocrinology of dominance: Basal testosterone predicts cortisol changes and behavior following victory and defeat. Journal of Personality and Social Psychology, 94(6), 1078.

Mehta, P. H., Wuehrmann, E. V., \& Josephs, R. A. (2009). When are low testosterone levels advantageous? The moderating role of individual versus intergroup competition. Hormones and Behavior, 56(1), 158-162.

Newman, M. L., Sellers, J. G., \& Josephs, R. A. (2005). Testosterone, cognition, and social status. Hormones and Behavior, 47(2), 205-211.

Oliveira, R. F. (2009). Social behavior in context: Hormonal modulation of behavioral plasticity and social competence. Integrative and Comparative Biology, icp055.

Oliveira, G. A., \& Oliveira, R. F. (2014). Androgen responsiveness to competition in humans: The role of cognitive variables. Neuroscience \& Neuroeconomicss, 3 .

Oliveira, R. F., Silva, A., \& Canário, A. V. (2009). Why do winners keep winning? Androgen mediation of winner but not loser effects in cichlid fish. Proceedings of the Royal Society B: Biological Sciences, rspb-2009.

Oliveira, G. A., Uceda, S., Oliveira, T., Fernandes, A., Garcia-Marques, T., \& Oliveira, R. F. (2013). Threat perception and familiarity moderate the androgen response to competition in women. Frontiers in Psychology, 4.

Op de Macks, Z. A., Moor, B. G., Overgaauw, S., Güroğlu, B., Dahl, R. E., \& Crone, E. A. (2011). Testosterone levels correspond with increased ventral striatum activation in response to monetary rewards in adolescents. Developmental Cognitive Neuroscience, 1(4), 506-516.

Packard, M. G., Cornell, A. H., \& Alexander, G. M. (1997). Rewarding affective properties of intra-nucleus accumbens injections of testosterone. Behavioral Neuroscience, 111(1), 219.

Packard, M.G., Schroeder, J.P., \& Alexander, G.M. (1998). Expression of testosterone conditioned place preference is blocked by peripheral or intra-accumbens injection of alpha-flupenthixol. Hormones and Behavior, 34, 39-47.

Peper, J. S., van den Heuvel, M. P., Mandl, R. C., Pol, H. E. H., \& Van Honk, J. (2011). Sex steroids and connectivity in the human brain: A review of neuroimaging studies. Psychoneuroendocrinology, 36(8), 1101-1113.

Preacher, K. J., \& Hayes, A. F. (2004). SPSS and SAS procedures for estimating indirect effects in simple mediation models. Behavior Research Methods, Instruments, \& Computers, 36(4), 717-731.

Riad-Fahmy, D., Read, G. F., Walker, R. F., Walker, S. M., \& Griffiths, K. (1987). Determination of ovarian steroid hormone levels in saliva. An overview. The Journal of Reproductive Medicine, 32(4), 254-272.

Ronay, R., \& von Hippel, W. (2010). The presence of an attractive woman elevates testosterone and physical risk taking in young men. Social Psychological and Personality Science, 1(1), 57-64.

Roney, J. R., Lukaszewski, A. W., \& Simmons, Z. L. (2007). Rapid endocrine responses of young men to social interactions with young women. Hormones and Behavior, 52(3), 326-333.

Salvador, A., \& Costa, R. (2009). Coping with competition: Neuroendocrine responses and cognitive variables. Neuroscience \& Biobehavioral Reviews, 33(2), 160-170.

Sapolsky, R. M. (1991). Testicular function, social rank and personality among wild baboons. Psychoneuroendocrinology, 16(4), 281-293.

Schultheiss, O. C., \& Rohde, W. (2002). Implicit power motivation predicts men's testosterone changes and implicit learning in a contest situation. Hormones and Behavior, 41(2), 195-202.

Schultheiss, O. C., \& Stanton, S. J. (2009). Assessment of salivary hormones. In E. Harmon-Jones \& J. S. Beer (Eds.), Methods in social neuroscience (pp. 17-44). New York: Guilford Press.

Schultheiss, O. C., Wirth, M. M., Torges, C. M., Pang, J. S., Villacorta, M. A., \& Welsh, K. M. (2005). Effects of implicit power motivation on men's and women's implicit learning and testosterone changes after social victory or defeat. Journal of Personality and Social Psychology, 88(1), 174.

Sellers, J. G., Mehl, M. R., \& Josephs, R. A. (2007). Hormones and personality: Testosterone as a marker of individual differences. Journal of Research in Personality, 41(1), 126-138.

Simmons, J. P., Nelson, L. D., \& Simonsohn, U. (2011). False-positive psychology undisclosed flexibility in data collection and analysis allows presenting anything as significant. Psychological Science, 22(11), 1359-1366.

Spielberger, C. D. (1983). Manual for the state-trait anxiety inventory STAI (form Y) (“ self-evaluation questionnaire"). 
Stanton, S. J., LaBar, K. S., Saini, E. K., Kuhn, C. M., \& Beehner, J. C. (2010). Stressful politics: Voters' cortisol responses to the outcome of the 2008 United States presidential election. Psychoneuroendocrinology, 35(5), $768-774$.

Touitou, Y., \& Haus, E. (2000). Alterations with aging of the endocrine and neuroendocrine circadian system in humans. Chronobiology International, 17(3), 369-390.

Trainor, B. C., Bird, I. M., \& Marler, C. A. (2004). Opposing hormonal mechanisms of aggression revealed through short-lived testosterone manipulations and multiple winning experiences. Hormones and Behavior, 45(2), 115-121.

van der Meij, L., Almela, M., Buunk, A. P., Fawcett, T. W., \& Salvador, A. (2011). Men with elevated testosterone levels show more affiliative behaviours during interactions with women. Proceedings of the Royal Society B: Biological Sciences, rspb20110764.

Watson, D., Clark, L. A., \& Tellegen, A. (1988). Development and validation of brief measures of positive and negative affect: The PANAS scales. Journal of Personality and Social Psychology, 54(6), 1063.

Wingfield, J. C., Hegner, R. E., Dufty Jr, A. M., \& Ball, G. F. (1990). The "challenge hypothesis": Theoretical implications for patterns of testosterone secretion, mating systems, and breeding strategies. American Naturalist, 829-846.

Wirth, M. M., Welsh, K. M., \& Schultheiss, O. C. (2006). Salivary cortisol changes in humans after winning or losing a dominance contest depend on implicit power motivation. Hormones and Behavior, 49(3), 346-352.

Zilioli, S., \& Watson, N. V. (2014). Testosterone across successive competitions: Evidence for a 'winner effect' in humans? Psychoneuroendocrinology, 47, 1-9.

Zilioli, S., Mehta, P., \& Watson, N. V. (2014). Losing the battle but winning the war: Uncertain outcomes reverse the usual effect of winning on testosterone. Biological psychology. 